University of Nebraska - Lincoln

DigitalCommons@University of Nebraska - Lincoln

Faculty Publications from the Department of Electrical \& Computer Engineering, Department Electrical and Computer Engineering

4-1-1991

\title{
Magneto-optical Kerr effect and perpendicular magnetic anisotropy of evaporated and sputtered Co/Pt multilayer structures
}

\author{
Ping $\mathrm{He}$ \\ University of Nebraska-Lincoln \\ William A. McGahan \\ University of Nebraska-Lincoln \\ John A. Woollam \\ University of Nebraska-Lincoln, jwoollam1@unl.edu \\ F. Sequeda \\ IBM Storage Systems Products Division, San Jose, California \\ T. McDaniel \\ IBM Storage Systems Products Division, San Jose, California \\ See next page for additional authors
}

Follow this and additional works at: https://digitalcommons.unl.edu/electricalengineeringfacpub

Part of the Electrical and Computer Engineering Commons

\begin{abstract}
He, Ping; McGahan, William A.; Woollam, John A.; Sequeda, F.; McDaniel, T.; and Do, H., "Magneto-optical Kerr effect and perpendicular magnetic anisotropy of evaporated and sputtered Co/Pt multilayer structures" (1991). Faculty Publications from the Department of Electrical and Computer Engineering. 55. https://digitalcommons.unl.edu/electricalengineeringfacpub/55
\end{abstract}

This Article is brought to you for free and open access by the Electrical \& Computer Engineering, Department of at DigitalCommons@University of Nebraska - Lincoln. It has been accepted for inclusion in Faculty Publications from the Department of Electrical and Computer Engineering by an authorized administrator of DigitalCommons@University of Nebraska - Lincoln. 


\section{Authors}

Ping He, William A. McGahan, John A. Woollam, F. Sequeda, T. McDaniel, and H. Do 


\title{
Magneto-optical Kerr effect and perpendicular magnetic anisotropy of evaporated and sputtered Co/Pt multilayer structures
}

\author{
Ping He, William A. McGahan, and John A. Woollam \\ Center for Microelectronic and Optical Materials Research, and Departments of Electrical Engineering \\ and Physics, University of Nebraska, Lincoln, Nebraska 68588-0511
}

F. Sequeda, T. McDaniel, and H. Do

IBM Storage Systems Products Division, San Jose, California 95193

(Received 14 November 1990; accepted for publication 2 January 1991)

\begin{abstract}
Thin and ultrathin $\mathrm{Co} / \mathrm{Pt}$ multilayered structures have been prepared on glass substrates by electron-beam evaporation at room temperature and by sputtering at various substrate temperatures and sputtering pressures. Perpendicular magnetic anisotropy was found in samples with $\mathrm{Co} / \mathrm{Pt}$ bilayer thicknesses near $3 \AA / 10 \AA$ and total thicknesses of the layer stack of no greater than $300 \AA$. X-ray diffraction was performed on the samples to determine layer spacing and integrity, and possible crystallinity of films. Crystalline structures in the interface between the Co and Pt layers were found and identified. The effects of sputtering parameters, such as pressure and substrate temperature, on the magneto-optical Kerr effect were studied. The two deposition methods, electron-beam evaporation and sputtering, resulted in different magneto-optical properties in samples with the same nominal layer structures. We have also investigated optical properties (reflectance, index of refraction, and extinction coefficient) of these materials using ellipsometry.
\end{abstract}

\section{INTRODUCTION}

Magneto-optical (MO) systems with compositionally modulated films have been the subject of numerous recent studies. ${ }^{1-4}$ Although some magneto-optical recording media using an amorphous rare-earth transition metal alloy (e.g., $\mathrm{TbFeCo}$ ) have recently reached the market place, there still exist problems, such as the easy oxidation of the rare-earth metal components, in these types of alloys. Metal multilayer systems, such as $\mathrm{Co} / \mathrm{Pd},{ }^{5,6} \mathrm{Co} / \mathrm{Pt}^{7-9}$ and $\mathrm{Co} / \mathrm{Ag}^{10}{ }^{10}$ with stable metal constituents, on the other hand, have several advantages and show some promise. The Co/Pt multilayer which has been studied by several groups is one of these new magneto-optical systems. ${ }^{7-9}$ Zeper et al. $^{8}$ and Carcia ${ }^{9}$ studied both evaporated and sputtered $\mathrm{Co} / \mathrm{Pt}$ multilayers on glass and $\mathrm{Si}$ substrates. The film thicknesses of their samples were from $500 \AA$ to a few thousand Angstroms, which are much thicker than the films prepared in the present experiments.

The magneto-optical Kerr effect (MOKE $=$ Kerr rotation $\theta_{k}$, and ellipticity $\epsilon_{k}$ ) in Co/Pt multilayer systems is more pronounced in the short-wavelength range. This characteristic does not correspond with the present wavelength range ( $>8000 \AA$ ) of lasers currently used for readout, but shorter wavelength lasers are under intensive development, and when implemented will allow high recording densities which are presently restricted by the diffraction limit of the light.

In this paper, we describe perpendicular magnetic anisotropy (PMA) in terms of the Kerr effect in evaporated and sputtered $\mathrm{Co} / \mathrm{Pt}$ multilayers, and show the MOKE dependence on the ratio of the Co-to-Pt layer thicknesses, on the total layer stack thickness, on the number of bilayers, and on sputtering parameters such as Ar gas pressure and substrate temperature. X-ray diffractograms are also presented, which reveal the rough interfaces in these compositionally modulated films, and the formation of $\mathrm{Co}-\mathrm{Pt}$ compounds at interfaces.

\section{EXPERIMENT}

The $\mathrm{Co} / \mathrm{Pt}$ multilayers were prepared by electronbeam evaporation at room temperature and by sputtering at three substrate temperatures $\left(25,50\right.$, and $\left.150^{\circ} \mathrm{C}\right)$ and at three sputtering pressures $(2.5,5.0$, and $8.5 \mathrm{mtorr})$. The multilayers were deposited onto glass substrates. Structurally, evaporated samples divided into three groups: E-I, E-II, and E-III, and sputtered samples divided into two groups: S-I and S-II, as shown in Tables I and II. In the first group of evaporated samples, the Co layer thickness and total thickness of the multilayer were fixed, and the thickness of the Pt layer was varied. In the second group of evaporated samples, both the Co and Pt layer thicknesses were fixed, and the number of bilayers varied. In the third group of evaporated samples, the Pt layer thickness and the overall thickness of the multilayer were fixed, but the Co layer thickness varied. The first four sputtered samples which formed the first group of sputtered samples have the same layer structure $\mathrm{Co}(3 \AA) / \mathrm{Pt}(10 \AA) \times 23$. The differences between them are underlayer material, substrate temperature, and sputtering pressure, as shown in Table II. The rest of the sputtered samples formed the second group of sputtered samples, having a slightly different layer structure $[300 \AA \mathrm{Pt}$ underlayer plus $\mathrm{Co}(3 \AA) / \mathrm{Pt}(16 \AA) \times 16]$

"Research supported by National Science Foundation Grant No. DMR 8605367. 
TABLE I. Structures and parameters of evaporated samples (See the Note of Table II).

\begin{tabular}{|c|c|c|c|c|c|c|c|c|c|}
\hline \multirow[b]{2}{*}{ Sample No. } & \multicolumn{4}{|c|}{ Group E-I } & \multirow[b]{2}{*}{4} & \multirow[b]{2}{*}{1} & \multicolumn{2}{|c|}{ Group E-II } & \multirow[b]{2}{*}{4} \\
\hline & 1 & 2 & 3 & & & & 2 & 3 & \\
\hline $\mathrm{Co} / \mathrm{p}$ t bilayer & $3 / 4$ & $3 / 6$ & $3 / 8$ & & $3 / 15$ & $3 / 10$ & $3 / 10$ & $3 / 10$ & $3 / 10$ \\
\hline No. of bilayers & 43 & 33 & 27 & & 17 & 8 & 15 & 38 & 77 \\
\hline Total thickness & 307 & 301 & 297 & & 306 & 104 & 195 & 494 & 1001 \\
\hline$H c(\mathrm{kOe})$ & 1.30 & 1.59 & 1.74 & & 1.36 & 1.69 & 1.80 & 1.80 & 3.77 \\
\hline \multirow[t]{2}{*}{$\theta_{k}$ (degrees) } & 0.26 & 0.25 & 0.20 & & 0.13 & 0.30 & 0.25 & 0.16 & 0.16 \\
\hline & \multicolumn{9}{|c|}{ Group E-III } \\
\hline Sample No. & 1 & 2 & & 3 & & & 5 & 6 & 7 \\
\hline Co/Pt bilayer & $1 / 10$ & $2 / 10$ & & $3 / 10$ & & & $5 / 10$ & $6 / 10$ & $7 / 10$ \\
\hline No. of bilayers & 27 & 25 & & 23 & & & 20 & 19 & 18 \\
\hline Total thickness & 297 & 300 & & 299 & & & 300 & 304 & 306 \\
\hline$H c(\mathrm{kOe})$ & 0.25 & 1.82 & & 1.88 & & & 0.73 & 0.58 & 0.54 \\
\hline$\theta_{k}$ (degrees) & 0.06 & 0.16 & & 0.21 & & & 0.22 & 0.24 & 0.26 \\
\hline
\end{tabular}

from those in first sputtered-sample group S-I. The sputtering parameters for these samples are also shown in Table II. In this way, optical and magneto-optical characterization of $\mathrm{Co} / \mathrm{Pt}$ multilayers were systematically studied.

MOKE (both Kerr rotation $\theta_{k}$ and ellipticity $\epsilon_{k}$ ) were measured with a rotating analyzer magneto-optical spectrometer discussed in detail elsewhere. ${ }^{11}$ The typical accuracy of the system is $\pm 0.005^{\circ}$ which satisfies most of the experimental requirements. The MOKE measurements were made over the $3000-8000 \AA$ spectral range, in a $0- \pm 8 \mathrm{kG}$ magnetic field with the light beam at a near normal incident angle of about 2 degrees. The MOKE hysteresis loops were measured at a wavelength of $5000 \AA$. Optical measurements were performed on a variable angle spectroscopic ellipsometer over the $3000-8000 \AA$ range at incident angles from $60^{\circ}-70^{\circ} .{ }^{12}$ Refractive index $n$, extinction coefficient $k$, and reflectivity of the films were determined from the measured ellipsometric parameters $\psi$ and $\Delta$ which are related to the Fresnel reflection coefficients $\widetilde{r}_{p}$ and $\widetilde{r}_{s}$ by the following equation:

$$
\widetilde{r}_{p} / \widetilde{r}_{s}=\tan (\psi) e^{i \Delta} \text {, }
$$

where $\widetilde{r}_{p}$ and $\tilde{r}_{s}$ are the $p$ - and $s$ components of the incident light beam, respectively. X-ray characterization was made with a Rigaku diffractometer, using $\mathrm{Cu} K_{\alpha}$ radiation $(\lambda=1.542 \AA)$.

\section{RESULTS AND DISCUSSION}

The integrity and periodicity of the sample layered structures were revealed by $\mathrm{x}$-ray diffraction. A typical low-angle $x$-ray diffractogram (sample E-III-7, evaporated) is shown in Fig. 1. The broad peak implies the lack of well defined interfaces between the individual layers. The position of the peak at $2 \theta \cong 5^{\circ}$ indicates that the bilayer thickness, or artificial period, is $17.5 \AA$ which is in good agreement with the nominal thickness of $17 \AA$. The $\mathrm{x}$-ray diffractograms in Figs. 2 and 3 show that for these samples the films are partially crystalline. The four diffractograms in Fig. 2 are from evaporated samples E-III-1, E-II-2, E-III-5, and E-III-7. The structures of these samples can be found in Table I. It is clear that the $\mathrm{X}$-ray peak moved from $2 \theta=41.6^{\circ}$ for sample E-III-7 to $2 \theta=40.5^{\circ}$ for sample E-III-1. The peak for sample E-III-7 indicates a $d$ spacing of $2.17 \AA$ which is exactly the $d$ spacing of tetragonal CoPt (101). ${ }^{13}$ When the Co layers in the films become thinner, the $d$ spacing changes to $2.22 \AA$ showing that the alloy $\mathrm{CoPt}$ transferred to cubic $\mathrm{CoPt}_{3}$ with $d(111)=2.22 \AA^{13}$ Therefore, the interfacial layers in the films are either the alloy CoPt for Co-to-Pt layer thickness ratio greater than about 7:10, or the alloy $\mathrm{CoPt}_{3}$ for the ratio less than or equal to 3:10. However, the structure of the interfacial layers at ratios between $3: 10$ and 7:10 is unknown. The $d$

TABLE II. Structures and parameters of sputtered samples. Note: The Co and Pt layer thicknesses are in Angstroms. $H c=$ coercivity. $\theta_{k}=\mathrm{Kerr}$ rotation at $\lambda=5000 \AA$. Spt. $P=$ Sputtering Pressure. Subst. $T=$ Substrate Temperature.

\begin{tabular}{|c|c|c|c|c|c|c|c|c|}
\hline \multirow[b]{2}{*}{ Sample No. } & \multirow[b]{2}{*}{1} & \multicolumn{2}{|c|}{ Group S-I } & \multirow[b]{2}{*}{4} & \multirow[b]{2}{*}{1} & \multicolumn{2}{|c|}{ Group S-II } & \multirow[b]{2}{*}{4} \\
\hline & & 2 & 3 & & & 2 & 3 & \\
\hline Underlayer & none & \multicolumn{7}{|c|}{$300 \AA \mathrm{Pt}$ for rest of the samples } \\
\hline Spt. $P$ (mtorr) & 2.5 & 2.5 & 8.5 & 2.5 & 2.5 & 5.0 & 8.5 & 8.5 \\
\hline$\theta_{k}$ (degrees) & 0.289 & 0.261 & 0.193 & 0.246 & 0.175 & 0.143 & 0.141 & 0.149 \\
\hline
\end{tabular}




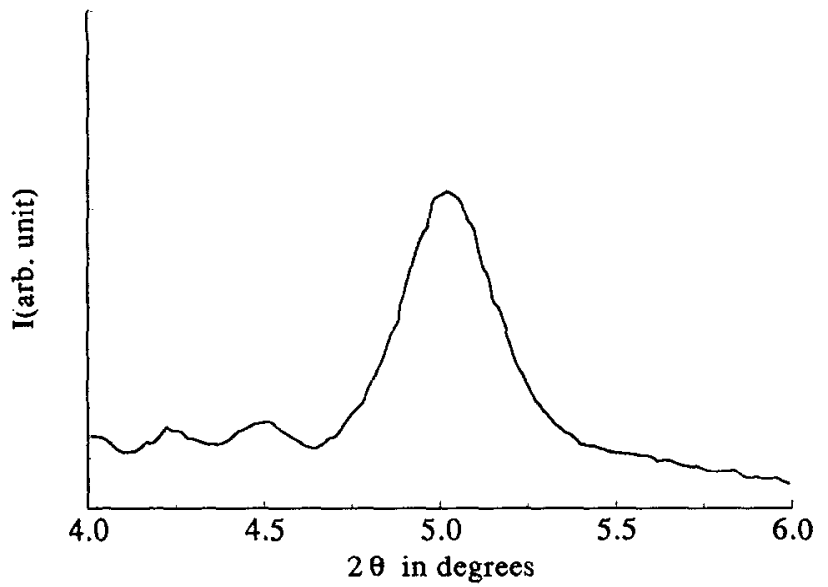

FIG. 1, X-ray diffractogram of Co/Pt compositionally modulated films of sample E-III-7. The peak indicates a layered structure of artificial period $17.5 \AA$.

spacing for fcc-Pt(111) is $2.266 \AA$ which showed up only in diffractograms from sputtered samples with $P t$ underlayers $(300 \AA)$. Therefore, there are not likely Pt crystals in the multilayer regions of films. The two diffractograms in Fig. 3 are of sputtered samples S-I-1 and S-I-2. The extra peak at about $2 \theta=39.5^{\circ}$ in Fig. 3 is from the underlayer platinum. Other $\mathrm{x}$-ray diffraction peaks are visible only in the diffractogram of sputtered samples S-II-4, as shown in Fig. 4. These peaks were identified as $\operatorname{Pt}(111)$, $\mathrm{CoPt}_{3}(111)$, fcc-Co(111), $\mathrm{CoPt}_{3}(200)$, and $\mathrm{Pt}(200)$ as labeled in Fig. 4. Consequently, most of the samples in these experiments contained cubic $\mathrm{CoPt}_{3}$ with a (111) texture oriented perpendicular to the films at the interface. The moments of $\mathrm{Co}$ and $\mathrm{Pt}$ are ferromagnetically coupled in both $\mathrm{CoPt}$ and $\mathrm{CoPt}_{3}$. Magnetic structures and properties of both $\mathrm{CoPt}$ and $\mathrm{CoPt}_{3}$ have been studied. ${ }^{14-16}$ The magnetic moment is $1.64 \mu_{B}$ for $\mathrm{Co}$ and $0.26 \mu_{B}$ for $\mathrm{Pt}$ in $\mathrm{CoPt}_{3}$, and $1.6 \mu_{B}$ for Co and $0.3 \mu_{B}$ for $\mathrm{Pt}$ in CoPt, respectively.

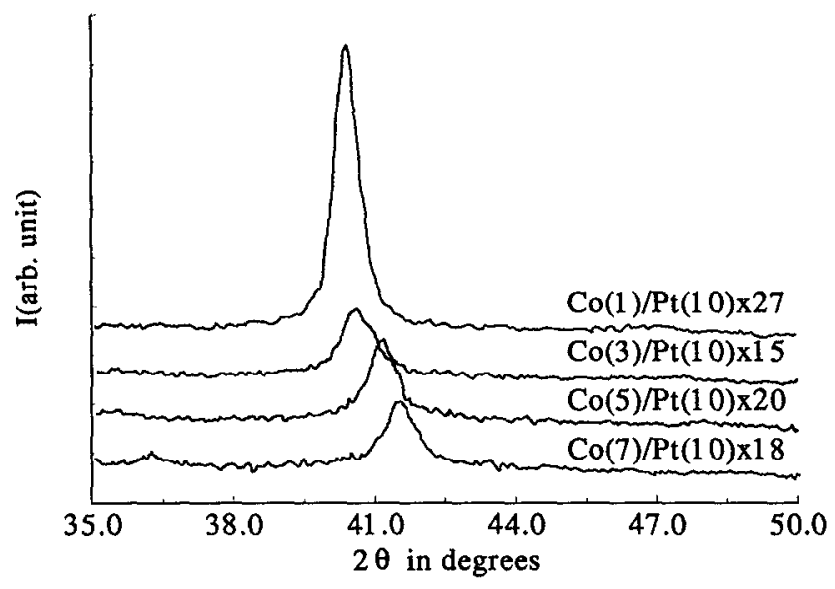

FIG. 2. X-ray diffractogram of $\mathrm{Co} / \mathrm{Pt}$ compositionally modulated films of sample E-III-1, E-II-2, E-III-5, and E-III-7. The shift of peaks means different phase of Co-Pt alloy.

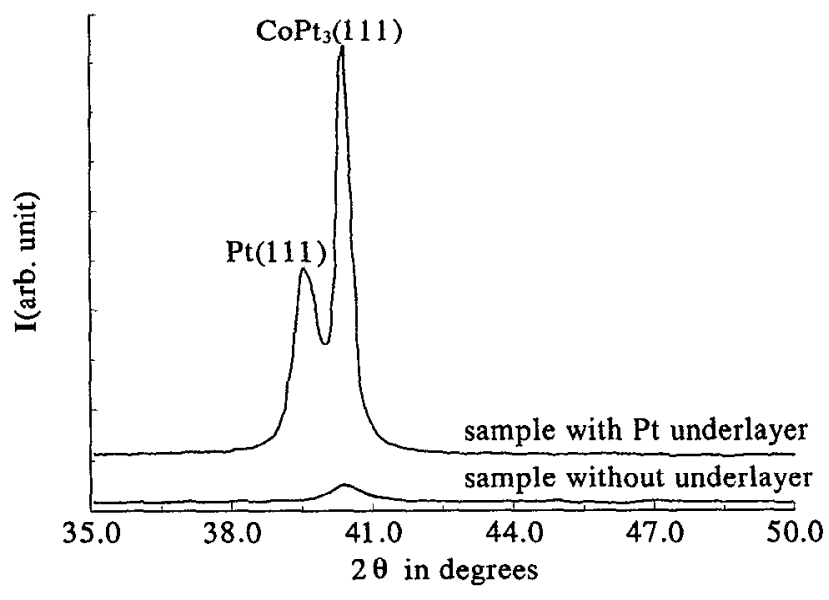

FIG. 3. X-ray diffractograms of sputtered samples S-I-1 without underlayer and S-I-2 with 300- $\AA \mathrm{Pt}$ underlayer.

Kerr rotations of evaporated samples listed in Table I are shown in Figs. 5-7. The Kerr rotation changed systematically with change in layer thickncsses of $\mathrm{Co}$ and $\mathrm{Pt}$, as shown in these figures. The Co-rich samples in groups E-I and E-III, such as samples E-I-1, E-III-6, and E-III-7, showed the largest Kerr rotations, but didn't have perpendicular magnetic anisotropies (PMA). For the Pt-rich sample E-III-1, the Kerr rotation was very small, and there was no PMA as well. The samples having large Kerr rotations and PMA are samples E-II-1 and E-II-2 as shown in Figs. 6 and 8. The structures of the two samples are: $\mathrm{Co}(3) / \mathrm{Pt}(10)$ with 8 bilayers for sample E-II-1 and $\mathrm{Co}(3) / \mathrm{Pt}(10)$ with 15 bilayers for samples E-II-2. Sample group E-II actually shows the effect of the total stack thickness of the multilayers. Figure 6 shows that the Kerr rotation for samples E-II-1 and E-II-2 which have stack thicknesses of 100 and $200 \AA$, respectively are much larger than the Kerr rotation for samples E-II-3 and E-II-4 with film thicknesses of about 500 and $1000 \AA$ (see Table I). It can also be seen from Fig. 9 (which shows Kerr rotation versus film thickness) that the Kerr rotation changes rap-

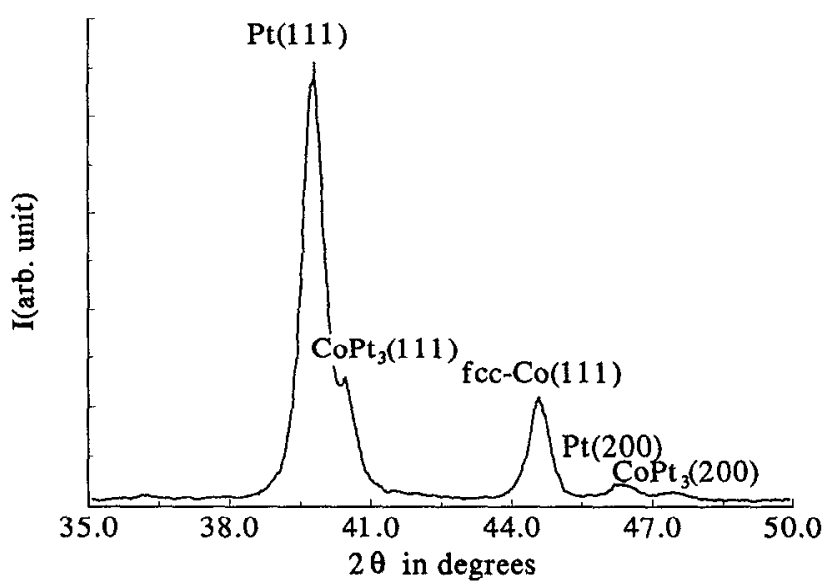

FIG. 4. X-ray diffractogram of sputtered sample S-II-4. More structures showed up for this sample. 


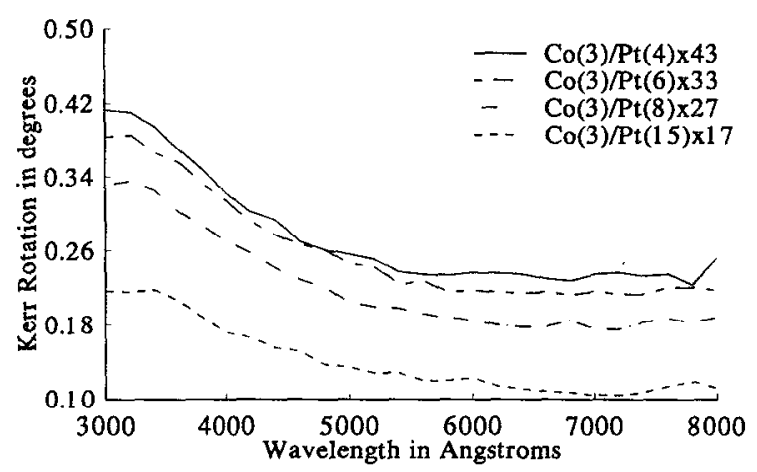

FIG. 5. Kerr rotations of sample group E-I.

idly when the films are thin. The results in Fig. 9 were selected from samples E-II-1, E-II-2, E-III-3, E-II-3, and E-II-4 which have the same Co-to-Pt layer thickness ratio $\mathrm{Co}(3 \AA) / \mathrm{Pt}(10 \AA)$ but different film thickness. The rapid change of the Kerr rotation when the films are thin may be due to the optical reflection from the glass substrate. On the other hand, the Kerr rotation from thick films is more intrinsical. In addition, the Kerr rotation of sample E-II-1 is almost constant over the entire spectral range of 3000 $8000 \AA$ A.

The individual layer effect on the MOKE spectra of evaporated $\mathrm{Co} / \mathrm{Pt}$ multilayer structures is shown in Figs. 7 and 10 where the Kerr rotation and MO hysteresis loops for sample group E-III are summarized (the sample E-III-6 loop is similar to the sample E-III-7 loop and is not shown). All samples in group E-III have the same film thickness $\sim 300 \AA$. The differences between the samples are the Co layer thickness and the number of bilayers. The more $\mathrm{Co}$ in the multilayers, the larger the Kerr rotation, as shown in Fig. 5. On the other hand, too much Co causes the films to lose PMA. The individual layer effects on the MOKE of electron beam evaporated $\mathrm{Co} / \mathrm{Pt}$ multilayers have been studied by other groups ${ }^{8,9}$ whose samples have different layer thicknesses.

Figure 11 shows the effect of the number of layers of PMA. The Kerr rotation loops in the figure were selected

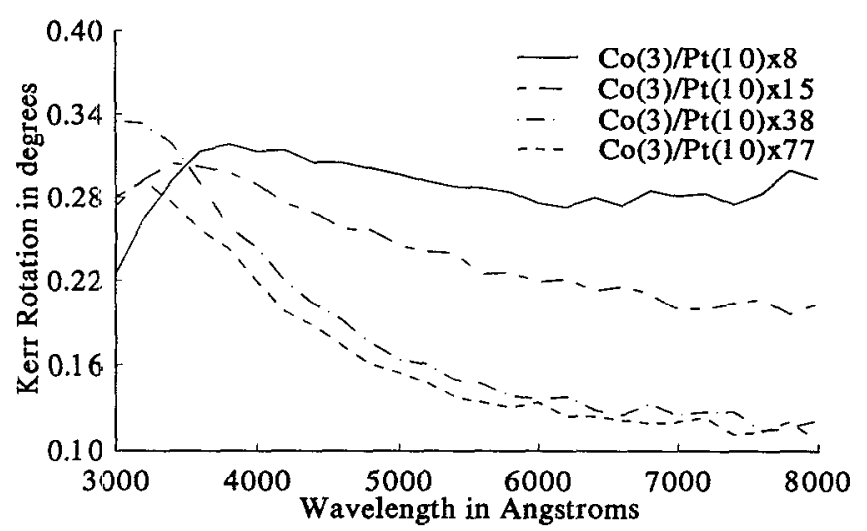

FIG. 6. Kerr rotations of sample group E-II.

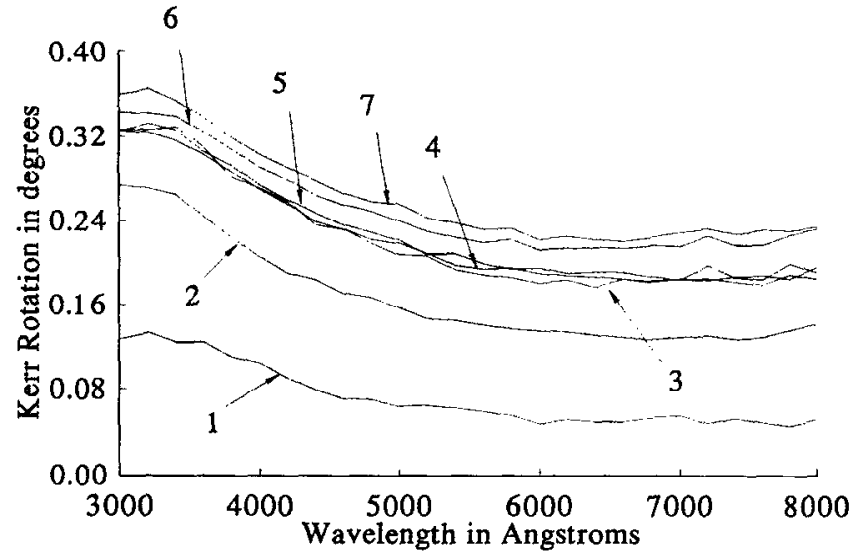

FIG. 7. Kerr rotations of sample group E-III. 1: E-III-1; 2: E-III-2; 3: E-III-3; 4: E-III-4; 5: E-III-5; 6: E-III-6; 7: E-III-7.

from evaporated sample groups E-I and E-III in such a way that the two loops in the same row were measured on samples which have the same Co-to-Pt layer thickness ratio and film thickness, but different numbers of bilayers [sample E-I-3 has a slightly different Co-to-Pt ratio(1:2.67) from sample E-III-4(1:2.5)]. The ones on the left have more bilayers than the ones on the right. It can be clearly seen that Kerr rotation loops on the left are broader than those on the right. Other than that, the two in the same row have almost the same shape. Therefore, the more bilayers, the higher the coercivity. Since the samples with more bilayers have more interfaces, or more interactions (couplings) between adjacent $\mathrm{Co}$ and $\mathrm{Pt}$ layers, it can be concluded that the magnitude of coercivity is correlated to the number and/or volume of Co and Pt layer interfaces, or the Co-Pt alloy. This point is also demonstrated in the results of evaporated sample group E-II in which sample E-II-1 with 8 bilayers has a coercivity $1.69 \mathrm{kOe}$ while the coercivity of sample E-II-4 with 77 bilayers is as high as $3.77 \mathrm{kOe}$. On the other hand, the number of bilayers has little effect on the Kerr rotation loop squareness of films.
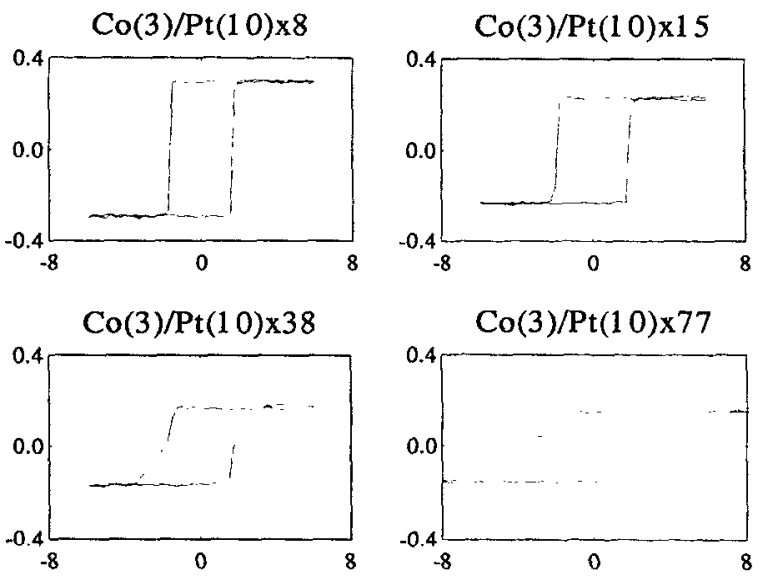

FIG. 8. MO hysteresis loops for sample group E-II. The $x$ axes are magnetic fields in $\mathrm{kG}$; the $y$ axes are Kerr rotations in degrees.

Ping He et al. 


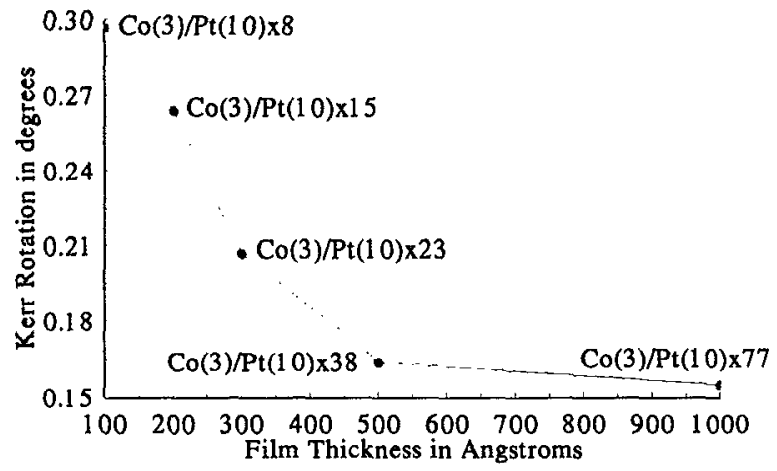

FIG. 9. Kerr rotation as a function of film thickness. The results are from samples E-II-1, E-II-2, E-III-3, E-II-3, and E-II-4 at $\lambda=5000 \AA$.

The present experiments show that sputter deposition results in different MO properties of films, and the sputtering pressure has a greater effect on the $\mathrm{MO}$ properties than does the substrate temperature. Figures 12 and 13 show that the Kerr rotation of sputtered films (sputtered S-I-1) is greater than that of evaporated films (sample E-III-3), but the coercivity of sputtered films is much smaller than the coercivity of evaporated films (see Tables I and II). Since the sputtered and evaporated films in this experiment had the same nominal layer structure, the different coer-
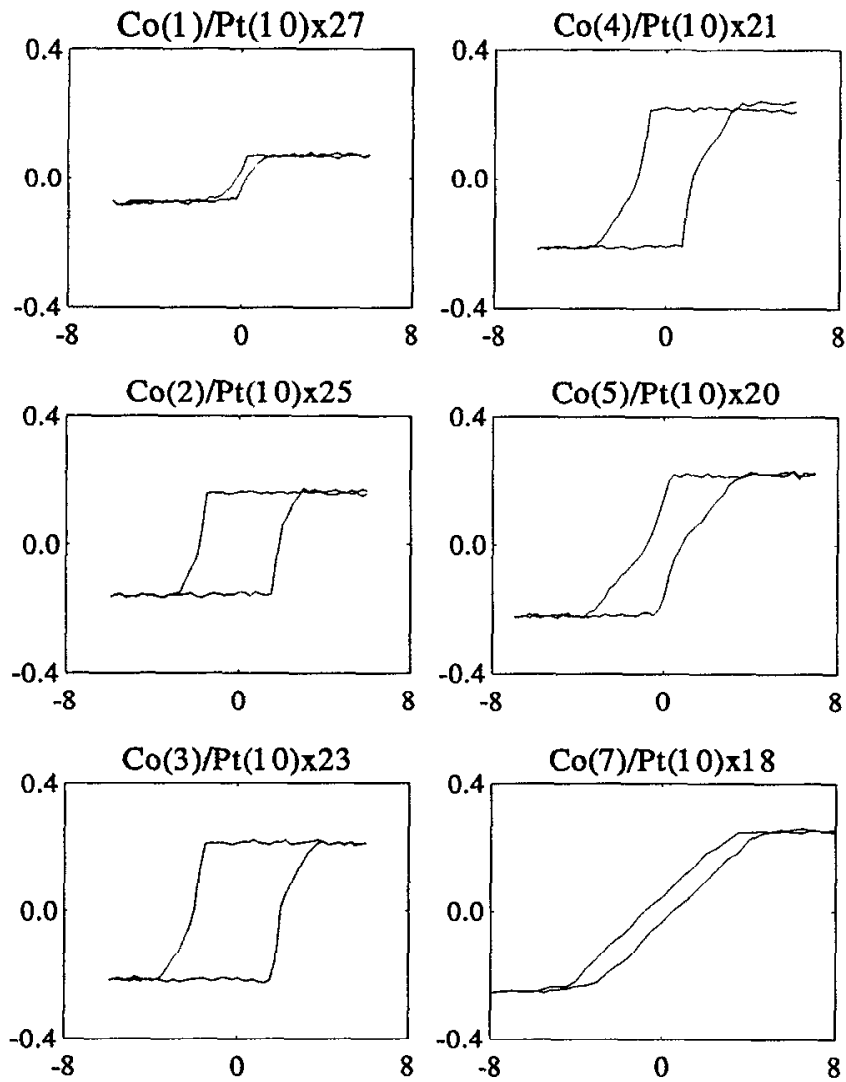

FIG. 10. MO hysteresis loops for sample group E-III. The $x$ axes are magnetic fields in $\mathbf{k G}$; the $y$ axes are Kerr rotations in degrees.
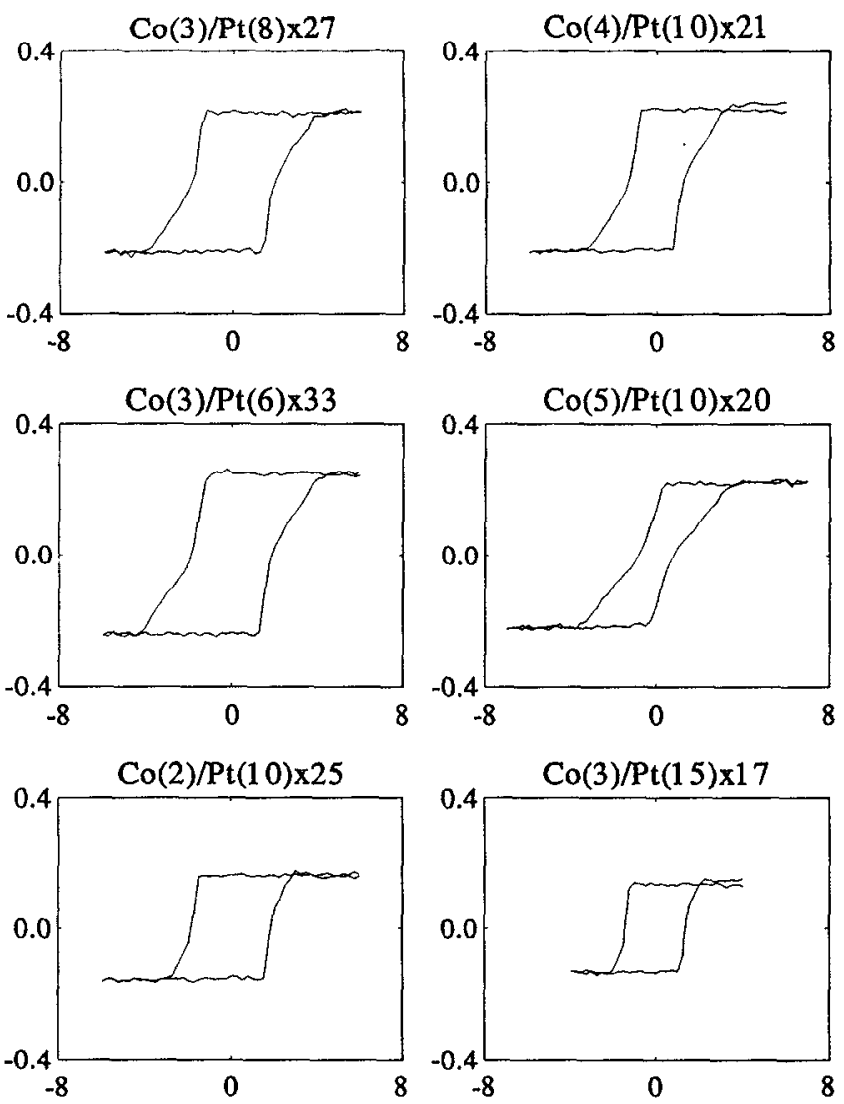

FIG. 11. MO hysteresis loops for selected samples for group E-I and E-III. The $x$ axes are magnetic fields in $\mathrm{kG}$; the $y$ axes are Kerr rotations in degrees.

civities may result from the different size, and/or different shape of grains or domains in the films. The sputtering pressure effect on the MOKE is shown in Figs. 14 and 15 where the results are from sputtered samples S-II-1, S-II-2, and S-II-3. Although the Kerr rotation increased almost 0.1 degrees by reducing the sputtering pressure from 5.0 to 2.5 mtorr, the coercivity of the film decreased from 800 to $300 \mathrm{Oe}$. On the other hand, increasing the sputtering pres-

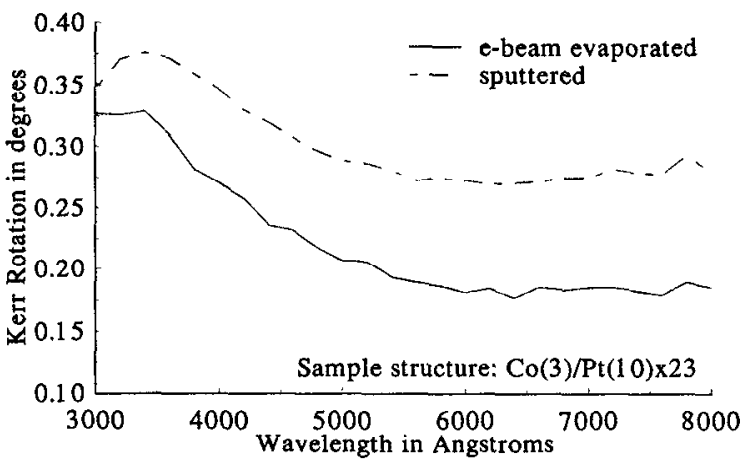

FIG. 12. The Kerr rotations of evaporated sample E-III-3 and sputtered sample S-I-1. 
Sputtered
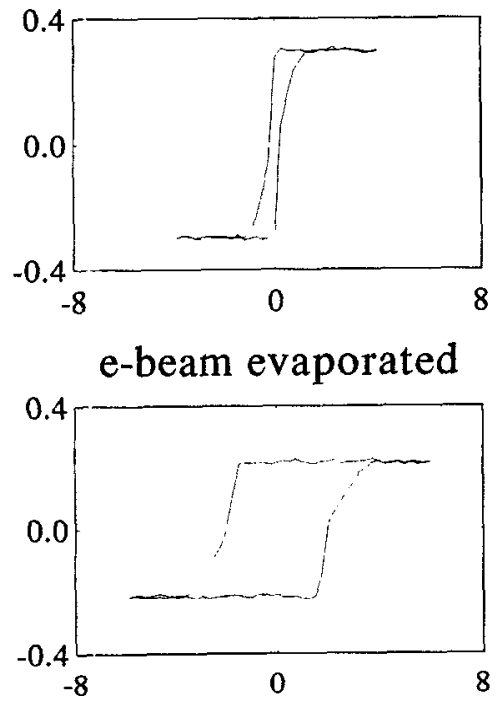

sure from 5.0 to $8.5 \mathrm{mTorr}$ didn't change the Kerr rotation, but almost doubled the coercivity (see Table II). In general, the higher the sputtering pressure, the lower the deposition rate and the less chances that the film is bombarded by Ar ions in the process of deposition. In lowsputtering-pressure cases, the reflected Ar ions from targets will hit or bombard the film on substrate and thus damage the surface of the film because of long mean free path. This bombardment of ions can result in small-size grains or domains on the surface of the film, and the film may show superparamagnetic properties. On the other hand, the situation of high-pressure sputtering is close to that of e-beam evaporation which involves no ion bombardments at all, and reasonably large grains or domains in the film are expected. Experiments with TEM and SEM need to be done to examine and to determine the size of grains and domains.

The underlayer coating had an interesting effect on the Kerr ellipticity. As shown in Fig. 16, the Kerr ellipticity of sputtered sample S-I-1 without underlayer coating is positive while the Kerr ellipticity of sputtered sample S-I-2

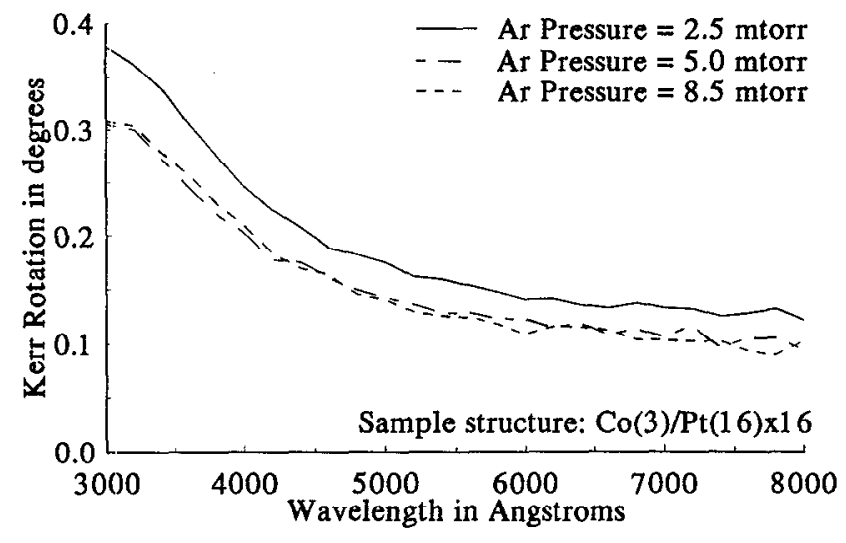

FIG. 14 Sputtering pressure effect. The Kerr rotations of sputtered samples S-II-1, S-II-2, and S-II-3.
Sputtered 5
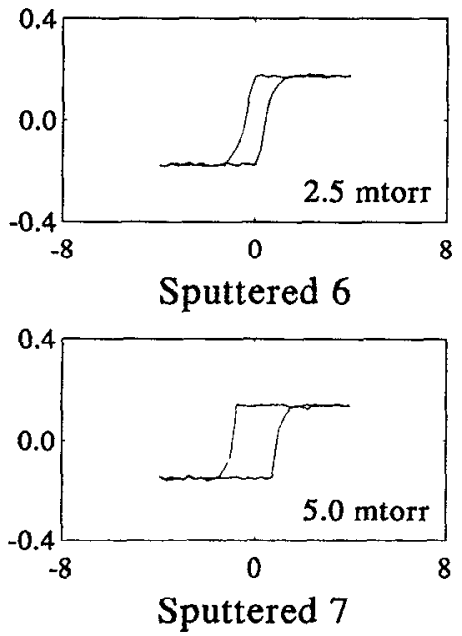

FIG. 15. Sputtering pressure effect. MO hysteresis loops of sputtered samples S-II-1, S-II-2, and S-II-3. The $x$ axes are magnetic fields in $\mathrm{kG}$; the $\boldsymbol{y}$ axes are Kerr rotations in degrees. The structure of the samples is $\mathrm{Co}(3$ Å) $/ \operatorname{Pt}(16 \AA) \times 16$.

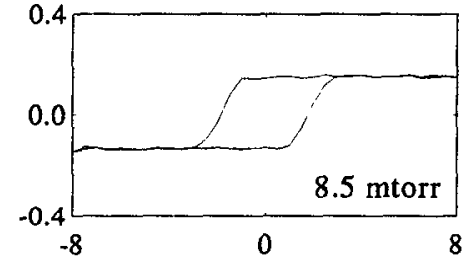

with an underlayer of $300 \AA \mathrm{Pt}$ is negative. As a matter of fact, all sputtered samples with underlayer coatings in this experiment had negative Kerr ellipticities. Since the films are not optically thick, the Pt underlayer plays a part of reflector and will have some effect on the MOKE, but the detail of the phenomenon is not clear. The substrate temperature was also varied from room temperature $\left(25^{\circ} \mathrm{C}\right)$ to $150^{\circ} \mathrm{C}$ as a parameter, but no significant changes were found in the MOKE.

The reflectance $R$ and optical constants $n$ and $k$ ( $n$ : refractive index and $k$ : extinction coefficient) of the samples were determined from the measured ellipsometric data, and the results for evaporated samples in group E-II are shown in Fig. 17. The optical constants $n$ and $k$ of bulk $\mathrm{Co}$ and $\mathrm{Pt}$ are also plotted in the same figure for comparison. ${ }^{16}$ As expected, the optical constants of the films are similar to those of bulk Co or Pt. Reflectances $R$, optical

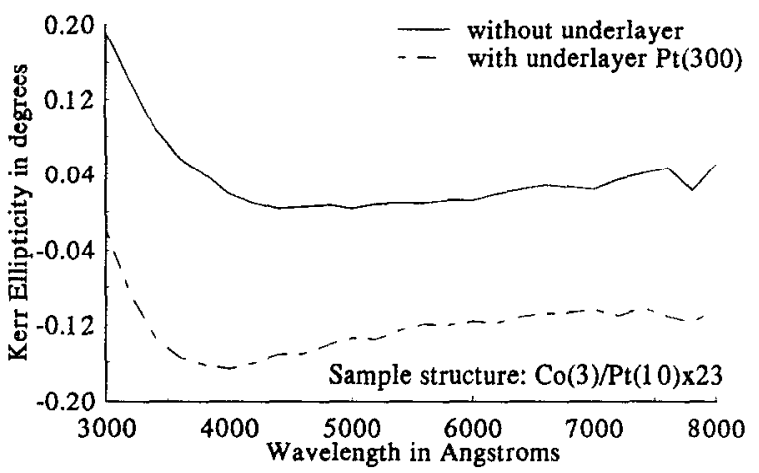

FIG. 16. Underlayer effect on the sign of the Kerr ellipticity. The Kerr ellipticity of sputtered samples S-I-1 and S-I-2. 
constants $n$, and $k$ of samples in evaporated sample groups E-I, E-III, and sputtered samples are very close to those of samples E-II-3 and E-II-4. Sample E-II-1 has a fairly low reflectance $(0.35-0.5)$. But because of its large Kerr rotation $\left(0.3^{\circ}\right)$, a respectable figure of merit $R\left(\theta_{k}\right)^{2}$ can still be expected, as shown in Fig. 18.

\section{CONCLUSION}

We have shown that $\mathrm{Co} / \mathrm{Pt}$ compositionally modulated films on glass substrates made by electron beam evaporation at room temperature and by sputtering at various substrate temperatures and sputtering pressures are promising materials for $\mathrm{MO}$ recording. The $\mathrm{Co} / \mathrm{Pt}$ films have both perpendicular magnetic anisotropy and a large Kerr rotation for a Co layer thickness of about $3 \AA$, Pt layers of
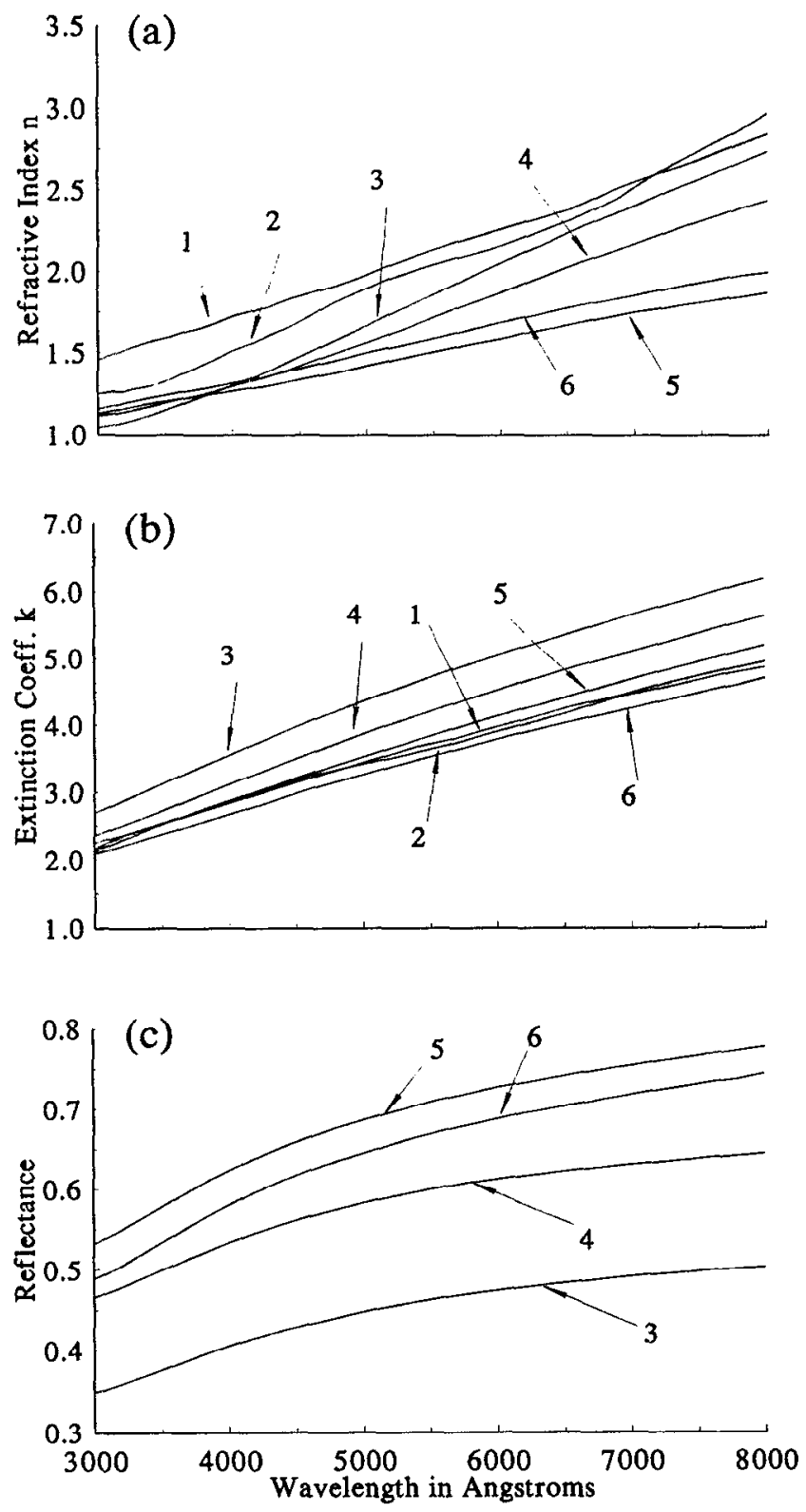

FIG. 17. (a) Index of refraction $n$, (b) extinction coefficient $k$, and (c) reflectance of samples in sample group E-II. 1: bulk Pt; 2: bulk $\mathrm{Co}$; 3 : sample E-II-1; 4: sample E-II-2; 5: sample E-II-3; 6: sample E-II-4. See Table I for the structures of these samples.

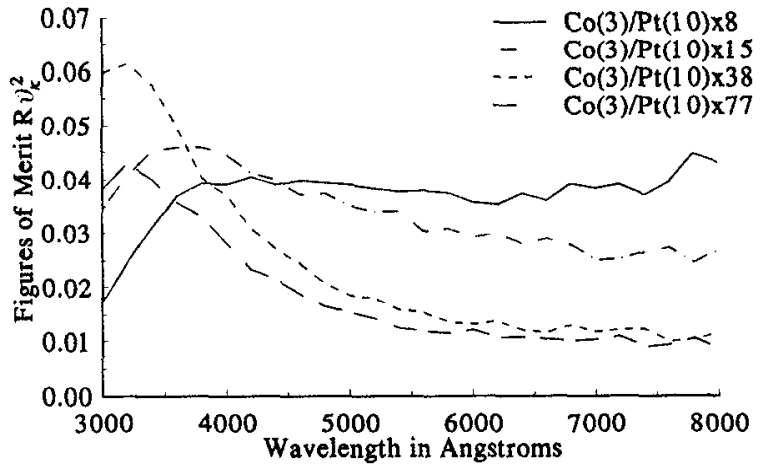

FIG. 18. Figures of merit $R \theta_{k}^{2}$ of evaporated samples in sample group E-II.

about 10 - $\AA$ thickness, and total thicknesses of the films of no greater than $300 \AA$. For most of the samples, the Kerr rotations are modest $\left(0.1^{\circ}-0.2^{\circ}\right)$ in the long wavelength range, but they reach maxima of $0.4^{\circ}-0.5^{\circ}$ at short wavelengths. The reflectances of the samples were estimated to be fairly high at long wavelengths $(70 \%-80 \%)$. Therefore, the figure of merit is still respectable, although the Kerr rotations are not especially large over the long wavelength spectral range. X-ray diffraction revealed that the interlayer structure between the Co and $\mathrm{Pt}$ layers is an alloy of either CoPt or $\mathrm{CoPt}_{3}$, depending upon the Co-to-Pt layer thickness ratio. For the layer thickness ratio less than $1: 3$, it is most likely that the compound $\mathrm{CoPt}_{3}$ is formed. It is also found that the number of bilayers affects the coercivity. The more layers in the films, the higher the coercivity. The sputtering pressure had a great effect on the MOKE, especially the MO hysteresis loops. The bombardments of Ar ions on the film surface may change the grain/domain size and shape, thus changing the coercivity of the film. The substrate temperature in the range from room temperature to $150^{\circ} \mathrm{C}$ had no effect on the MOKE. Comparison of electron-beam evaporation method with sputtering deposition method showed that different MO properties of films will be expected for the same nominal structure.

There are still open questions concerning the physics of the $\mathrm{Co} / \mathrm{Pt}$ layered systems, especially the roles played by $\mathrm{Co}$ and $\mathrm{Pt}$ chemistry and microstructure in the interlayers. Also, the coercivity correlated to the number/nature of the interfaces of the Co and $\mathrm{Pt}$ layers need further investigations. Because both $\mathrm{Co}$ and $\mathrm{Pt}$ are very stable metals, MO properties of our samples have not changed over a period of about three months, while exposed to laboratory atmosphere.

${ }^{1}$ G. A. N. Connell, J. Magn. Magn. Mat. 54-57, 1561 (1986).

${ }^{2}$ P. Hansen, M. Hartmann, and K. Witter, J. Magn. Soc. Jpn., Vol. II, Supplement, No. S1, 257 (1987).

${ }^{3}$ Y. J. Choe, S. Tsunashima, T. Katayama, and S. Uchiyama, J. Magn.

Soc. Jpn., Vol. II, Supplement, No. S1, 273 (1987).

${ }^{4}$ D. Weller and W. Reim, Appl. Phys. A 49, 599 (1989).

${ }^{5}$ D. G. Stinson and S. C. Shin, J. Appl. Phys. 67, 4459 (1990).

${ }^{6}$ S. Hashimoto, Y. Ochiai, and K. Aso, J. Appl. Phys. 66, 4909 (1989).

${ }^{7}$ G. Schütz, R. Wienke, W. Wilhelm, W. B. Zeper, H. Ebert, and K. Spörl, J. Appl. Phys. 67, 4456 (1990). 
${ }^{8}$ W. B. Zeper, F. J. A. M. Greidanus, P. F. Carcia, and C. R. Fincher, J. Appl. Phys. 65, 4971 (1989).

${ }^{9}$ P. F. Carcia, J. Appl. Phys. 63, 5066 (1988).

${ }^{10}$ R. Kurishnan, V. Cagan, M. Porte, and M. Tessier, J. Magn. Magn. Mat. 83, 65 (1990).

${ }^{11}$ L. Y. Chen and J. A. Woollam, SPIE 1166, 267 (1989).

${ }^{12}$ S. A. Alterovitz, J. A. Woollam, and P. G. Snyder, Solid State Technol. 31, 99 (1988).
${ }^{13}$ Any standard $\mathrm{x}$-ray diffraction handbook.

${ }^{14}$ B. Van Laar, J. de Physique 25, 600 (1964).

${ }^{15}$ F. Menzinger and A. Paoletti, Phys. Rev. 143, 365 (1966).

${ }^{16}$ R. M. Bozorth, Ferromagnetism, (Van Nostrand, New York, 1951).

${ }^{17}$ J. H. Weaver, C. Krafka, D. W. Lynch, and E. E. Koch, Physik Daten: Optical Properties of Metals (Fach-information-zentrum, Karlsruhe, 1981). 